\title{
Le religieux dans la sociologie allemande
}

Des classiques aux questions contemporaines : autres références, autres perspectives?

Anne-Sophie Lamine et Hubert Knoblauch

\section{OpenEdition}

12 Journals

Édition électronique

URL : http://journals.openedition.org/assr/26094

DOI : 10.4000/assr.26094

ISSN : $1777-5825$

Éditeur

Éditions de l'EHESS

\section{Édition imprimée}

Date de publication : 20 octobre 2014

Pagination : $11-30$

ISBN : 978-2-7132-2433-1

ISSN : 0335-5985

Référence électronique

Anne-Sophie Lamine et Hubert Knoblauch, «Le religieux dans la sociologie allemande », Archives de sciences sociales des religions [En ligne], 167 | juillet-septembre 2014, mis en ligne le 20 octobre 2017, consulté le 19 avril 2019. URL : http://journals.openedition.org/assr/26094; DOI : 10.4000/assr.26094 


\section{Postérités allemandes Communication, transcendance, non-religion}





\section{Anne-Sophie Lamine Hubert Knoblauch}

\section{Le religieux dans la sociologie allemande Des classiques aux questions contemporaines: autres références, autres perspectives ${ }^{1}$ ?}

Pourquoi consacrer un dossier à la sociologie de la religion ${ }^{2}$ en Allemagne dans une revue francophone de sciences sociales des religions? Exercice difficile, qui sera forcément très imparfait par ses choix et ses oublis. Il nous a cependant semblé nécessaire et utile de le tenter, en partant de ces deux constats : l'héritage encore aujourd'hui très différencié des auteurs classiques (anciens ou modernes) et la méconnaissance des travaux contemporains de sociologie allemande des religions par le public francophone ${ }^{3}$. Les textes rassemblés ici entendent contribuer modestement à combler ces manques. D’emblée, un article concerne directement un auteur classique par excellence, Georg Simmel dont les réflexions sur la religion demeurent encore relativement peu connues des lecteurs francophones. Le texte choisi est une présentation originale de son approche du religieux par un spécialiste reconnu (Volkhard Krech). Ce dossier offre ensuite les traductions inédites de deux auteurs "classiques contemporains ${ }^{4}$ ", Niklas Luhmann et Thomas

1. Nous remercions Pierre Lassave pour la relecture attentive de l'ensemble du dossier et Patrick Watier pour celle de cet article.

2. Le terme allemand étant Religionssoziologie nous avons choisi de garder « religion » au singulier dans cet article, à la différence de la formulation française actuelle qui utilise plus fréquemment le pluriel («des religions » in D. Hervieu-Léger et J.-P. Willaime, 2001, J.-P. Willaime, 1995, O. Bobineau et S. Tank-Storper, 2007 ; " de la religion » in R. Cipriani, 2004).

3. Cette relative méconnaissance ne doit évidemment pas nous faire sous-estimer le rôle des passeurs entre ces deux cultures. Parmi les sociologues français germanophones contemporains ayant contribué à ces passages, par des recensions, des articles, des traductions et des chapitres d'ouvrages collectifs, on peut mentionner Jean-Paul Willaime, Jean Baubérot, Claire de Galembert, Freddy Raphael, Nikola Tietze, Patrick Watier (voir par exemple le collectif M. Koenig et J.-P. Willaime (dir.), 2008). En remontant un peu en arrière, il faut notamment signaler les nombreuses recensions d'ouvrages allemands de Religionssoziologie par Jean Séguy pour les Archives de Sociologie des Religions.

4. Ces deux auteurs sont considérés comme des " classiques" (ou "nouveaux classiques " ou "classiques contemporains») en sociologie allemande. Par exemple l'ouvrage de présen- 
Luckmann, encore peu connus des sociologues francophones du fait religieux, alors qu'ils ont produit des textes déterminants dans ce domaine mais dont aucun (relevant de cette thématique) n'était jusqu'alors disponible en français. En ce qui concerne les recherches contemporaines, les travaux sociologiques sur le religieux se rejoignent à bien des égards de part et d'autre du Rhin, comme plus largement au sein du continent européen, que l'on pense aux débats sur la sécularisation, sur l'individualisation de l'expérience religieuse ou encore sur les nouvelles dynamiques religieuses. À cet égard, ce dossier propose trois thématiques qui nous apparaissent comme des contributions spécifiques et originales de la sociologie allemande des religions, pour des raisons à la fois contextuelles (historiques et contemporaines) et intellectuelles. La première est le " tournant communicationnel » en sociologie des religions (Hubert Knoblauch), qui a été développé par des chercheurs à la fois spécialistes de Luckmann et marqués par les sociologies habermassienne et luhmannienne. La deuxième thématique est une sociologie des non-religieux, dont le développement spécifique a évidemment été poussé par la situation spécifique de l'ex-RDA et de son héritage historique induisant une forte prévalence de l'athéisme quasi institutionnel et de l'indifférence religieuse. Celle-ci est abordée de manière qualitative en comparant l'exRDA et les USA (Monika Wohlrab-Sahr et Tom Kaden) puis quantitative, en " testant » la théorie de la sécularisation à partir de données européennes (Detlef Pollack). Ces diverses positions mènent naturellement à la troisième thématique : une ou des modernités ? Le concept de modernités multiples est mis à l'épreuve d'une approche néo-institutionnaliste (Matthias Koenig). On peut là encore faire l'hypothèse que la situation spécifique de l'Allemagne au sein de l'Europe a favorisé cette attention à la pluralité des modernités (ou sécularités), à la fois par sa bi-confessionnalité historique, par l'inclusion récente des nouveaux Länder de l'Est et par sa proximité géographique avec l'aire culturelle orthodoxe.

\section{Mise en perspective historique}

Un bref regard historique sur le domaine thématique considéré permet de placer le cadre, et de poser quelques points de comparaison avec le cas français. En Allemagne, comme dans d'autres contextes nationaux, une sociographie des pratiques religieuses a d'abord été portée par l'attention des Églises au détachement religieux des couches populaires et du monde urbain en expansion. Cette sociologie pastorale s'est développée après la Seconde Guerre mondiale. Cependant, l'intérêt sociologique pour la religion est bien antérieur. Les fondateurs

tation, Thomas Luckmann, écrit par Bernt Schnettler (2006) dans la collection « Klassiker des Wissensoziologie " (les classiques de la sociologie de la connaissance) ou le chapitre sur Luhmann (Rudolf Stichweh, 1999) dans le Klassiker der Soziologie. Von Talcott Parsons bis Pierre Bourdieu, de Dirk Kaesler. Karl Gabriel and Hans-Richard Reuter considèrent Luckmann et Luhmann (ainsi que P. Bourdieu et P. Berger) comme des " néo-classiques ", dans leur manuel, Religion und Gesellschaft. Texte zur Religionssoziologie (2004). 
allemands les plus fameux de la discipline, Georg Simmel et Max Weber, ont aussi été de fins observateurs de la religion, et sont internationalement reconnus parmi les fondateurs de la sociologie des religions (Hartmann Tyrell et al., 1995). La religion a été l'objet d'une critique normative et socialement informée de Marx et de ses disciples (H. Tyrell, 1995). Weber a notamment souligné la nécessité de laisser de côté les jugements de valeur sur les sujets d'étude sociologique. Il a même confessé son manque d' "oreille musicale " à propos du religieux. Mais contrairement aux sociologues français, notamment durkheimiens, Simmel et plus encore Weber insistent sur la dimension subjective de la religion. Le contenu psychologique impliqué dans la «religiosité » simmelienne s'oppose à la forme culturelle objectivée de la religion. Du côté wébérien, cela prend la forme d'une orientation subjective vers un monde invisible "caché ", et d'une insistance sur le rôle du charisme et des idées religieuses comme orientations générales pour les acteurs ${ }^{5}$.

À la différence de la France où l'esquisse d'un programme sociologique a été tracée par Auguste Comte dès le milieu du XIX e siècle et dont l'institutionnalisation démarre avant la fin du siècle grâce à Durkheim, la sociologie allemande s'institutionnalise pendant la période de la République de Weimar, bien que le premier Soziologentag ${ }^{6}$ ait eu lieu dès 1910. Mais, tout comme en France, les débuts de la sociologie allemande de la religion sont lents ${ }^{7}$. Dans les années 1920, Max Scheler consacre quelques pages particulièrement profondes à la religion, dans le cadre de ses écrits sur la sociologie de la connaissance, une nouvelle sous-discipline qui se développe alors (1993 [1926] : 113-133). Quelques années plus tard, Joachim Wach ${ }^{8}$ (1898-1955) consacre une importante monographie à la sociologie des religions (1931, traduite en français en 1955). Il émigre aux États-Unis peu après, en 1935. Son parcours est tout à fait emblématique du sort national de la discipline universitaire, le triomphe du fascisme et de la terreur nazie provoquant l'émigration de nombreux savants. Le parcours intellectuel de Wach est aussi caractéristique du style de la sociologie de la religion allemande pendant ces décennies. Inspiré à la fois par l’herméneutique, la phénoménologie

5. D. Goldschmidt (1959a, 1959b) inclut aussi l'historien et théologien Ernst Troeltsch comme l'un des fondateurs de la sociologie de la religion.

6. La société des sociologues allemands avait été créée l'année précédente. Lors de ce premier congrès des sociologues allemands, Weber et Simmel ont d'ailleurs engagé un débat sur la sociologie de la religion, suite à l'exposé de Troeltsch (P. Watier, 2008 : 206).

7. Pour une approche synthétique des débuts de la sociologie des religions en France, voir : D. Hervieu-Léger, 1998 ; J.-P. Willaime, 1995 : 40-55, 1999, 2004 ; C. de Galembert, 2004 ; A. Mary, 2006, ainsi que les premiers numéros des Archives de Sociologie des Religions ; pour les débats des années 1950 aux années 1980 au sein de la CISR, O. Tchannen, 1990 ; pour des perspectives internationales : J. Beckford, 2000 et G. Davie, 2004.

8. Pour une présentation concise de Wach en langue française: R. Cipriani (2004: 204-207) ainsi qu'un dossier qui lui est consacré dans le premier numéro des Archives de Sociologie des Religions avec un notamment un hommage de son successeur à l'université de Chicago (J. Kitagawa, 1956) et une discussion de sa sociologie (H. Desroche, 1956). 
et la sociologie compréhensive, il contribue à préparer le terrain pour les études empiriques de la religion tout en devenant membre de la Divinity School de l'Université de Chicago. On peut encore mentionner le professeur pronazi Gustav Mensching (1901-1978), qui suit l'approche de Wach ainsi que de Rudolf Otto et publie une Religionssoziologie systématique en 1947 (traduite en français en 1951). Il s'investit ensuite non dans la sociologie, mais dans la science de la religion (Religionswissenschaft). Cette dernière, d'abord établie au sein des départements de théologie, devient une discipline indépendante à partir des années 1920, alors que la sociologie de la religion devient - et demeure - une partie de la Religionswissenschaft, tout comme l'histoire et la phénoménologie de la religion.

Le monde intellectuel germanophone a été marqué comme on l'a vu par de nombreux départs vers les États-Unis. La New School for Social Research joue alors un rôle essentiel de refuge pour nombre d'intellectuels juifs ou d'origine juive fuyant le nazisme, comme Alfred Schütz (1899-1959), Albert Salomon (1891-1966), Aron Gurwitsch (1901-1973) ou encore Carl Mayer (1902-1974) dont l'épouse est juive ${ }^{9}$. Reinhard Bendix (1916-1991) fera sa thèse à l'université de Chicago. La période d'après-guerre est celle d'un véritable tournant pour la sociologie. Les idées, et parfois les hommes, retraversent l'Atlantique. Ce n'est qu'à partir de ce moment-là que Max Weber va véritablement être reconnu comme un auteur classique en Allemagne. Cela passe par la lecture des travaux d'auteurs américains ou émigrés qui l'ont considéré comme un des pères fondateurs de la sociologie : ceux de Talcott Parsons, de Reinhard Bendix ou encore de Carl Meyer (1933), qui fut par ailleurs aussi un des professeurs de Thomas Luckmann. Comme ailleurs en Europe, l'influence des auteurs américains, et notamment de leurs approches empiriques est grande aussi dans les divers domaines de la sociologie ${ }^{10}$. La sociologie de la religion adapte les méthodes empiriques de Gabriel Le Bras $(1955,1956)$ ainsi que celles d'auteurs américains. Dans un rapport présenté au congrès allemand de sociologie à Berlin en $1959^{11}$, sur l'état de la sociologie de la religion, Dietrich Goldschmidt (1959a, b) explique qu'à ce moment-là, la recherche empirique sur la religion est menée dans trois types

9. Sur le rôle de la New School, voir C. Schrecker (dir.), (2012); S. Lymann (1994); sur le rôle des migrations intellectuelles, J. Heilbron et al., 2009.

10. Voir R. Münch (1991) sur la domination de la sociologie américaine après guerre (accentuée en Allemagne par l'idée que la pensée sociale aurait eu une part de responsabilité), mais aussi sur la remobilisation des traditions européennes, qui vont se décliner selon les aires nationales : marxisme et structuralisme en France, dialectique de la modernité (théories critiques et systémiques) en Allemagne ; voir aussi S. Kalberg (2004 : 181-182). En retraçant la « crise [contemporaine] de la critique sociale » de part et d'autre du Rhin, D. Trom pointe une autre différence significative : "la raison est objet de réalisation dans l'histoire, dans la tradition allemande, elle coïncide avec la science en France » (2008: 109).

11. Ce texte est publié dans les actes du congrès (1959a), ainsi que - fait intéressant - très rapidement et en allemand, dans les Archives de Sociologie des Religions (1959b), ce qui témoigne de l'intérêt de plusieurs des animateurs de la revue, qui recensent aussi les ouvrages importants de sociologie de la religion en langue allemande. 
de centres : les centres de recherche non-confessionnels, les instituts relevant de facultés de théologie des deux confessions ainsi que certains instituts ecclésiaux. Il évoque aussi la toute nouvelle "renaissance » de la sociologie de la religion, au cours de ces années 1950, en mentionnant notamment la création d'une section qui se constitue au sein de la société allemande de sociologie en 1957.

Un « remigrant » va jouer un rôle particulier dans le renouvellement du domaine thématique dans l'Allemagne d'après-guerre. C'est le sociologue Thomas Luckmann, qui a fait ses études à Vienne, et qui est parti aux États-Unis après la guerre. Il se forme auprès d'Alfred Schütz et de Carl Mayer à la New School. Il y rencontre aussi Peter L. Berger. Après un mémoire de master sur Albert Camus, dirigé par Schütz, il se voit proposée une place d'assistant à la chaire de Mayer pour une enquête sur le développement de communautés ecclésiales dans l'Allemagne d'après-guerre (B. Schnettler, 2006 : 26-28) et soutient une thèse de sociologie en $1956^{12}$. Quelques années plus tard, il écrit un article très critique à l'égard de la sociologie ecclésiale de la religion publié dans la Kölner Zeitschrift für Soziologie (1960). Il y affirme que la sociologie de la religion s'est considérablement appauvrie en restreignant la religion à sa forme institutionnelle incarnée par les Églises. Il développe ensuite sa critique dans un essai de 84 pages, publié en allemand (1963). Ce second texte est la première version de ce qui va devenir Invisible Religion. The Problem of Religion in Modern Society (New York, 1967), ouvrage classique dans de larges sphères linguistiques ${ }^{13}$, mais pas dans le monde francophone. Dans l'intervalle, Luckmann publie avec Berger, The Social Construction of Reality (1966 [La Construction sociale de la réalité, 1986]) qui deviendra le manifeste fondateur du « constructivisme social » et aujourd'hui un ouvrage "classique-contemporain » de sociologie. C'est suite à cela que l'université de Francfort lui propose un poste (où il collabore, entre autres, avec Jürgen Habermas), puis, en 1970, l'université de Constance, où se déroule la fin de sa carrière et son éméritat.

L'intérêt grandissant pour cette perspective, qui envisage le religieux de manière plus large et plus constructiviste est attesté par nombre de publications innovantes ainsi que par l'International Yearbook for Sociology of Knowledge and Religion, qui est publié en anglais et en allemand ${ }^{14}$. Il y eut cependant une

12. Pour une interview de Luckmann en français, sur son parcours, voir T. Luckmann et J. Ferreux, 2006.

13. La version américaine de l'ouvrage est traduite en Italien (1969), espagnol (1973) et japonais (1976). En 1991 elle est retraduite en allemand et fait l'objet de compléments (1991). La version allemande de 1991 est traduite en polonais (1996), chinois (1997), slovène (1997), alors que des extraits sont publiés dans divers Textbooks en langue anglaise, allemande, néerlandaise et italienne.

14. L'Internationales Jahrbuch für Religionssoziologie, paraît de 1965 à 1973 puis devient Internationales Jahrbuch für Wissens und Religionssoziologie, de 1973 à 1978. Parmi les autres publications collectives, signalons K.-F. Daiber et T. Luckmann (dir.), 1983. 
dispute intellectuelle de courte durée à la fin des années 1970, juste avant la publication de la Funktion der Religion de Luhmann (1982) qui causa une interruption dans la publication du Yearbook et la dissolution de la section de sociologie de la religion, qui n'a été refondée qu'en 1995. Il n'est pas surprenant que la conférence de redémarrage ait porté sur "La religion comme communication » (H. Tyrell et al., 1995), dans la mesure où la communication, dans l'intervalle, était au centre des approches les plus innovantes au sein des théories sociales allemandes avec notamment les apports de Luckmann, Luhmann et Habermas ${ }^{15}$. L'importance de la sociologie de la religion a continué à croitre, notamment après le 11 septembre 2001, lorsque la religion est érigée en sujet médiatique. Bien que le nombre de professeurs d'université en sociologie de la religion en Allemagne (ou dans l'espace germanophone) soit particulièrement bas (que ce soit en science des religions ou en sociologie), cette sous-discipline est devenue particulièrement créative, en terme de recherche empirique et de développements théoriques.

On n'est pas surpris qu'un article de synthèse sur l'évolution de la sociologie allemande de la religion (M. Wohlrab-Sahr, 2000) s'intitule "What has happened since "Luckmann 1960"? ". L'auteure y brosse un aperçu large de la production germanophone pendant ces quatre décennies, en parcourant successivement les héritages des anciens classiques: Weber et Simmel, les apports et discussions des classiques contemporains: Luhmann et Luckmann, puis thématiquement, les travaux sur la sécularisation et l'individualisation, sur l'indifférence religieuse (inspirés par le cas de l'ex-RDA) et pour finir le débat entre sociologie de la religion et sociologie des Églises qui recoupe largement le débat entre quantitavistes et qualitativistes et la réduction du religieux à la participation aux activités d'une Église (voir aussi M. Koenig et C. Wolf, 2013).

Au-delà de ce bref survol historique, on peut s'interroger sur ce qui est finalement spécifique aujourd'hui dans la Religionssoziologie ? Précisons d'abord que, comme en France, la culture scientifique souffre de la délégitimation croissante de la langue nationale pour la communication scientifique, au profit de l'anglais (même si ce problème est légèrement atténué en France par l'existence d'une communauté francophone dépassant les frontières de l'hexagone). Bien qu'elle soit fortement influencée par des auteurs américains et français, dont beaucoup sont traduits, la sociologie de l'espace germanophone est principalement publiée en allemand (on peut estimer la proportion à plus de $90 \%$ ). En dépit d'une tendance croissante à l'internationalisation et aux collaborations ${ }^{16}$, la sociologie de la religion en Allemagne est allemande, car elle est construite sur un discours

15. Ces trois auteurs ont très fortement influencé la sociologie allemande en général et la sociologie de la religion en particulier (Habermas, dans une moindre mesure pour la seconde, et Luhmann, bien plus que les deux autres auteurs pour l'une et l'autre).

16. Le réseau thématique de recherche "Sociology of Religion » de l'European Sociological Association a été fondé par deux allemands (B. Schnettler, H. Knoblauch) et une française (A.-S. Lamine) en 2011. 
dense qui se restreint le plus souvent à l'espace germanophone. Le tournant communicationnel illustre parfaitement cela : il apparaît comme une spécificité du débat allemand depuis Habermas, Luhmann et Luckmann, ainsi que plus récemment avec le «constructivisme communicationnel ». Autre spécificité, à la différence du discours anglo-saxon, et dans une moindre mesure du cas français, la sociologie allemande de la religion a gardé des liens très étroits avec les débats théoriques en cours en sociologie et plus largement dans les sciences sociales. Cette large place accordée au débat théorique est d'ailleurs soulignée par plusieurs auteurs français, contemporains ou des débuts de la discipline, non sans parfois une pointe critique ${ }^{17}$. Ainsi Jean Séguy souligne-t-il en 1970 les points communs entre sociologues allemands et français, mais aussi leur écart concernant la théorisation :

Le phénomène a été dénoncé avec justesse et virulence par Thomas Luckmann [1963, qui a fait l'objet d'une recension]. [...] Nos Archives avaient ailleurs déjà agité le grelot [contre la sociologie d'Église] pratiquement depuis leur fondation, mais avec moins d'enthousiasme pour des théorisations généralisatrices. Henri Desroche était sans doute le seul dans ses Sociologies religieuses entre autres et non exclusivement à prêcher en faveur de points de vue globaux. Cependant, tous les sociologues le savent, et cela explique la discrétion de notre revue en ce domaine, la globalisation présente l'inconvénient, par son caractère même, de ne pouvoir présenter de garanties absolues quant au domaine de la preuve. D'où la nécessité d'accumuler les dossiers avant toute théorisation. Mais quand a-t-on assez récolté de données pour pouvoir généraliser? La question n'admet pas de réponse facile (J. Séguy, 1970, p. 100).

Plus récemment, Willaime a évoqué «la grande tradition théorique de la sociologie allemande»(1995, p. 50). Kalberg souligne aussi l'importance de la théorie dans la sociologie (générale) allemande, en retraçant son histoire et dans une perspective comparative avec la France et les États-Unis. La sociologie française partage avec sa cousine allemande ce lien continental avec la philosophie même si son dialogue avec l'anthropologie, la psychanalyse, l'histoire et la critique littéraire l'en ont quelque peu éloignée ${ }^{18}$ (S. Kalberg, 2004). En dépit de variations propres à chaque contexte national, il y a bien là un trait marquant qui distingue les deux sociologies, dont les articles présentés dans ce volume sont très largement emblématiques. Ce tropisme théorique va cependant de pair avec des développements innovants en méthodes qualitatives ${ }^{19}$.

17. Cette revendication moins affirmée du côté français ne signifie évidemment pas une moindre production (et circulation) de concepts en sociologie des religions, que l'on pense aux notions de "validation du croire ", d' " exculturation ", " religion en mode mineur " ou de " post-modernité ", pour n'en citer que quelques-unes.

18. Ce fait a été redoublé pour la sociologie des religions par son alliance institutionnelle avec l'anthropologie, au sein du CNRS (où elle est bien mieux représentée qu'à l'université, aussi bien à ses débuts que maintenant).

19. Qualitative Religionsforschung est la première monographie strictement dévolue au lien entre méthodologie qualitative et sociologie de la religion (H. Knoblauch, 2003). 
Une autre caractéristique spécifique du domaine germanique découle des relations spécifiques entre les institutions religieuses et la recherche académique ou les universités en général. On observe une présence forte de la théologie de plusieurs confessions dans des universités publiques allemandes ${ }^{20}$ (catholique romaine, luthérienne, réformée, musulmane, juive). Alors qu'au cours des dernières décennies de larges pans des sciences des religions se sont délibérément détachés de la théologie, la sociologie des religions semble plus ambivalente à cet égard. D'un côté, la recherche sociologique répondant à une demande ecclésiale est massive, supportée principalement par les deux grandes confessions qui jouent, comparativement à la France, un rôle bien plus important sur le plan politique et surtout économique. En conséquence, la recherche sociologique financée par les Églises est de plus en plus souvent menée au sein des départements universitaires de théologie ou au sein d'instituts ecclésiaux. Il y est même question d'une "Christliche Sozialwissenschaft ». D'autre part, s'il reste quelques postes académiques en sociologie de la religion, il n'y a pas d'institut universitaire spécialisé en la matière (même le nouvel Institut Max-Planck pour l'étude de la diversité ethnique et religieuse, fondé en 2007 à Göttingen, intègre la question religieuse dans celle de la migration).

Les positions institutionnelles recoupent partiellement le clivage scientifique. D'un côté, on envisage la religiosité comme principalement vécue dans un rapport à l'institution. De l'autre, on privilégie des approches plus ou moins constructivistes qui font place aux formes de religiosité hors des Églises. Bien qu'on ait, des deux côtés, accommodé l'argument de l'autre, l'écart entre les deux camps est significatif, à la fois théoriquement et méthodologiquement. La sociologie ecclésiale mise aussi sur les méthodologies d'enquêtes et sur la théorisation à moyenne portée, alors que la sociologie académique penche vers les méthodes qualitatives et les "grandes théories ». Ces clivages, cependant, se superposent maintenant aux débats sur la sécularisation, le retour du religieux et la relation entre politique et religion qui traversent les deux camps.

\section{Lisons-nous les mêmes "classiques " ?}

Un dossier sur la sociologie allemande de la religion invite à cette question : lisons-nous les mêmes classiques de part et d'autre du Rhin ? On a bien le sentiment que la réponse risque d'être négative, mais il s'agit de la préciser. Certes, malgré sa réception tardive en France, Weber est devenu un classique de la discipline en général et de la sociologie des religions en particulier, mais qu'en est-il des autres auteurs ? Une recherche à l'aide de "google scholar» - en dépit des

20. Rappelons qu'en France, le seul cas d'université publique incorporant des instituts de théologie (protestante et catholique) se trouve à Strasbourg, ville qui relève du régime concordataire propre au territoire d'Alsace et de Moselle. 
limites évidentes de la méthode - offre une première indication de la place des auteurs mentionnés dans les sociologies de la (des) religion(s) germanophone et francophone ${ }^{21}$. Elle donne les résultats suivants :

\begin{tabular}{|l|r|r|r|r|r|r|}
\hline $\begin{array}{c}\text { Auteur } \\
\text { Langue }\end{array}$ & Simmel & Weber & Durkheim & Luhmann & Luckmann & Bourdieu \\
\hline Allemand & 1380 & 5190 & 1980 & 2130 & 1500 & 1170 \\
\hline Français & 492 & 3660 & 2820 & 145 & 347 & 906 \\
\hline
\end{tabular}

Sans surprise, ces chiffres indiquent que Weber et Durkheim sont considérés comme les classiques les plus importants en sociologie des religions des deux côtés du Rhin. Ils font aussi apparaître que Simmel est dans une position qui semble plus secondaire - sans pour autant être négligé - en France, et que Luhmann et Luckmann (plus fréquemment cité dans ses travaux communs avec Berger) y sont relativement peu cités. L'importance de Luhmann dans le débat allemand peut s'expliquer par le fait que la théorie systémique s'est établie comme une sorte de discours intellectuel, même au-delà du monde académique, de manière comparable à la réception de Bourdieu en France. Bien que les textes de Bourdieu soient beaucoup moins mentionnés que ceux de Luhmann, ils constituent une référence significative des deux côtés. On peut encore souligner que le " collège de sociologie » français (G. Bataille, R. Caillois, 1937-1939) a eu un très faible écho du côté allemand ${ }^{22}$.

Une indication plus fine de l'importance accordée aux "classiques » peut être mise en évidence par l'analyse des ouvrages introductifs contemporains. En Allemagne, après la sociologie des religions de Wach parue en 1931, nombre de ces ouvrages introductifs sont édités après guerre. Les plus influents ${ }^{23}$ (maintenant ou dans le passé) semblent aussi correspondre à ceux qui ont offert une présentation systématique de la sociologie de la religion et de ses principaux résultats, tel celui de Matthes $(1967,1969)$ qui a été un sociologue de la religion des plus reconnus pendant plusieurs décennies. Actuellement, on peut mentionner trois monographies et un recueil de textes qui remplissent ce rôle. L'anthologie établie par Gabriel et Reuter (2004) commence, de manière intéressante,

21. Pour chaque auteur XX la recherche a été effectuée dans chaque langue : soit respectivement [("religionssoziologie" OR "soziologie der religion") AND XX] et [("sociologie des religions” OR “sociologie de la religion”) AND XX], en restreignant à la langue de recherche (recherche actualisée le 28/05/2014).

22. Voir cependant une étude récente sur les relations entre le Collège de Sociologie et la sociologie allemande (S. Moebius, 2006) alors que du côté français, ces approches sont souvent ignorées ou qualifiées d' "insolites " (E. Diantell et M. Löwy, 2009).

23. En plus des ouvrages présentés dans le texte, on peut aussi mentionner: G. Kehrer (1968), W. Stark (1974), B. Wallisch-Bärber (1977), J. Hach (1980), G. Kehrer (1988) et H.-J. Helle (1997). 
par un texte de Durkheim. Cependant, elle inclut aussi Simmel, Weber, et Troeltsch comme " classiques » et Luckmann, Berger, Luhmann et Bourdieu comme " néoclassiques "; les approches allemandes récentes sont représentées par des textes de Felix Kaufmann, Günter Dux (un élève de Luckmann), Ulrich Oevermann, Martin Riesebrodt et Monika Wohlrab-Sahr aux côtés d'Eileen Barker, Robert N. Bellah et José Casanova. Parmi les autres livres toujours disponibles chez leurs éditeurs, Krech (1999) propose une introduction, courte et accessible d'une centaine de pages, qui démarre avec Comte, Durkheim et Weber, se poursuit avec Luckmann et Luhmann, pour aborder enfin les thématiques de la globalisation et du New Age. Un autre ouvrage introductif, Knoblauch (1999), plus épais (250 pages) inclut en outre des chapitres sur Bourdieu, les questions de genre, les nouveaux mouvements religieux, les expériences religieuses - thème pertinent pour les nouvelles spiritualités. L'ouvrage le plus récent, Pickel (2011), présente deux différences : d'une part, parmi les « classiques ", seul Luhmann est envisagé après Marx, Durkheim, Weber et Simmel ; d'autre part, la préférence sociologique penche vers les méthodes quantitatives auxquelles deux chapitres sont consacrés. Si ces divers ouvrages ont en commun les mêmes auteurs classiques, ils se différencient par la place accordée aux auteurs de sociologie de la connaissance ainsi que dans leurs orientations théoriques et la part relative entre méthodologie qualitative et quantitative.

À titre de comparaison, voyons la place des classiques allemands dans les ouvrages introductifs à la sociologie des religions en France. Ces derniers sont de deux types : présentations des auteurs ou présentations combinées d'auteurs et de thématiques ${ }^{24}$. Les premiers sont des volumes plus épais (Hervieu-Léger et Willaime, 2001, Diantell et Löwy, 2005 et 2009 ; Cipriani, 2004) alors que les seconds sont en format de poche, d'une centaine de pages (Willaime J.-P., 1995, O. Bobineau et S. Tank-Storper, 2012). Danièle Hervieu-Léger et JeanPaul Willaime (2001) ont écrit un premier volume présentant les "classiques ", leur choix se portant sur huit auteurs, trois allemands et cinq français : Marx, Tocqueville, Weber, Simmel, Durkheim, Halbwachs, Le Bras et Desroche. Quelques années plus tard, l'anthropologue Erwan Diantell et le sociologue Michaël Löwy $(2005,2009)$ présentent dans un deuxième volume des approches qu'ils qualifient de «dissidentes » : Ernst Troeltsch, Karl Mannheim, Ernst Bloch, Antonio Gramsci, Marcel Mauss, Zora Neale Hurston, Roger Bastide, Lucien Goldmann et Pierre Bourdieu, puis « insolites ", dans une troisième livraison : W. E. B. Du Bois, Walter Benjamin, Erich Fromm, Michel Leiris, Roger Caillois, Lydia Cabrera, Ernesto De Martino, E. P. Thompson, Eric Hobsbawm. En dépit de cette large diversité, les approches de Luckmann et de Luhmann sont absentes de cet ensemble de trois volumes. Le Manuel du sociologue italien Roberto Cipriani, paru en 2004, se distingue nettement des autres introductions.

24. Pour une discussion plus large de manuels de poche récents en sociologie et anthropologie des religions, voir P. Lassave, 2008. 
Il prend le parti de la présentation de nombreux auteurs européens et nordaméricains (une soixantaine) et s'apparente donc davantage à une mini-encyclopédie de la sociologie des religions, unique en langue française. Il consacre plusieurs pages à Luckmann et à Luhmann. En ce qui concerne les manuels de poche, celui de Willaime (1995) a été plusieurs fois réédité, alors que le second (O. Bobineau et S. Tank-Storper, 2007) est plus récent. Ces ouvrages ont en commun la place importante donnée aux classiques (Marx, Tocqueville, Durkheim, Simmel, Weber, plus brièvement Luhmann et Luckmann pour le premier; Durkheim et Weber pour le second) et aux thématiques de la modernité, de la sécularisation et de la définition du religieux. Simmel n'est donc pas mentionné dans le second ouvrage, pas plus que Luhmann, mais Luckmann est traité pour son approche originale de la sécularisation. Willaime faisant partie des "passeurs " franco-allemands contemporains $^{25}$, il n'est pas surprenant qu'il accorde plus d'intérêt aux auteurs allemands. Les ouvrages français accordent une place importante aux classiques qui peuvent se limiter à Durkheim et Weber ou être bien plus nombreux. Globalement, on y accorde relativement peu d'importance (hormis les questions de définition de la religion et de la sécularisation) à la discussion des divers types de théorisation sociologiques - implicites ou explicites - du rapport à la religion (choix rationnel, socialisation, compensation, individualisation...), contrairement aux manuels allemands (H. Knoblauch, 1999) ou en langue anglaise (I. Furseth, P. Repstad, 2006 ; G. Davie, 2007).

Il faut cependant rappeler que la réception « lente et difficile » de la sociologie de Max Weber en France ne commence réellement qu'à partir de la fin des années $1960^{26}$. En ce qui concerne celle de Simmel, si certains textes sur la religion paraissent en français dès $1903^{27}$, sa réception reste assez faible en France, bien que plus accessible depuis la fin des années $1990^{28}$. Quant à Luckmann et Luhmann, comme on l'a souligné en introduction, les deux textes livrés dans ce volume sont les seuls - sur le religieux - traduits en français, à notre connaissance. En ce qui concerne la traduction d'auteurs contemporains, les passages entre les deux langues restent difficiles. Si la sociologie générale est bien traduite et introduite d'une langue à l'autre ${ }^{29}$, il en va bien autrement des sociologies de la religion,

25. Voir note 3 supra.

26. Raymond Aron en est le promoteur depuis les années 1930. L'éthique protestante est traduite par Jacques Chavy en 1964, et Le judaïsme antique par Freddy Raphaël en 1965. Alors que les autres textes de sociologie des religions ne seront traduits qu'au cours des années 1990. Sur la réception de Weber, voir M. Pollak (1988) et J.-P. Willaime (2001: 63-66).

27. Dans la Revue de Métaphysique et de Morale.

28. Maurice Halbwachs l'inclut certes dans ces cours de sociologie à Strasbourg dès 1919 mais Raymond Aron ne lui consacre que quelques pages dans sa Sociologie allemande de 1935. On doit à Jean Séguy, en 1964, une traduction de la première version (1906) de Die Religion dans les Archives de Sociologie des Religions. Le volume La Religion, incluant la traduction de l'essai éponyme (version longue) ainsi que deux autres textes, est édité en 1998, par Patrick Watier qui en a fait une présentation (1996), ainsi que J.-P. Willaime (2001: 111-146).

29. L'éditeur UVK a fait traduire Bourdieu, Kaufmann et d'autres sociologues français. Latour et Boltanski sont aussi rapidement traduits. La présentation générale de Moebius et 
séparées par un "mur d'ignorance»(I. Chiva, 1987, à propos de l'ethnologie). Dans un sens comme dans l'autre, les développements les plus récents ne sont connus que de quelques chercheurs, surtout s'ils ne sont pas publiés en anglais ${ }^{30}$.

\section{Du tournant communicationnel à l'analyse du non-religieux et des modernités multiples}

L'approche de Simmel se signale dans ce volume par un commentaire original de la notion de religiosité. Volkhard Krech nous montre qu'en plus d'être ce " rythme de l'intériorité » ou cette attitude caractérisée par des combinaisons "d'aspiration et de jouissance, de don et de reprise, d'humilité et d'exaltation, de fusion et de tenue à distance ", elle est aussi une forme d'ascétisme individuel, dirigé vers un but existentiel, dans une forme de conquête de soi. Krech nous montre aussi comment Simmel anticipe à sa manière les synthèses luhmannienne et luckmannienne entre individualité et socialisation. Face à l'inévitable tension entre culture subjective et culture objective, entre individualisation et socialisation, les codes religieux et l'expérience de la religiosité permettent de symboliser et de ressentir l'unité de l'existence. Dans ce sens, la religiosité est un seuil entre intériorité et socialisation.

La communication est devenue ces dernières décennies un concept central dans le débat théorique allemand, si bien qu'on parle de "tournant communicationnel $^{31}$ » de la sociologie allemande, dans lequel les pensées de Habermas, Luhmann et Luckmann jouent des rôles différents, mais essentiels ${ }^{32}$. Dans sa Théorie de l'agir communicationnel Jürgen Habermas emprunte des éléments à

Peter (2004) inclut : M. Crozier/E. Friedberg, R. Boudon, A. Touraine, P. Bourdieu, G. Balandier, E. Morin, J.-C. Kaufmann, B. Lahire, J. Baudrillard, M. Maffesoli, B. Latour, D. Schnapper, L. Boltanski/L. Thevenot, R. Castel. Dans l'autre sens, on a accès à beaucoup de traductions françaises de Luhmann, Habermas, Beck, Honneth, mais très peu de Joas. Les présentations générales de la sociologie allemande ne manquent pas, d'Aron à Papilloud en passant par Vandeberghe.

30. Le survol des auteurs français contemporains de sociologie des religions traduits en allemands est rapide : Danièle Hervieu-Léger (Le Pèlerin et le Converti, 1999 [Pilger und Konvertiten, 2004]), Olivier Roy (L'islam mondialisé, 2002 [Der islamische Weg nach Westen, 2006] et la Sainte Ignorance, 2008 [Heilige Einfalt, 2011], Nilufer Göle (Musulmanes et modernes. Voile et civilisation en Turquie, 1993 [Republik und Schleier. Die muslimische Frau in der modernen Türkei, 1995] et Interpénétrations. L'islam et l'Europe, 2005 [Anverwandlungen. Der Islam in Europa zwischen Kopftuchverbot und Extremismus, 2008]). On peut cependant aussi souligner, à titre de comparaison, que ni Grace Davie, ni James Beckford ne sont traduits en allemand. Des auteurs plus nombreux (F.-A. Isambert, J.-P. Willaime, C. de Galembert, etc.) sont présents dans des ouvrages collectifs.

31. Dans une perspective wébérienne et sur Habermas et Luhmann, voir W. Schluchter (2005 : 676-677) ; dans une perspective luckmannienne, voir H. Knoblauch (1995; 2001).

32. La communication n'est cependant pas absente de la sociologie française, notamment d'inspiration pragmatiste-interactionniste, comme l'atteste l'article lumineux de Quéré (1991). 
la pragmatique et au tournant linguistique selon Wittgenstein, tout en intégrant la théorie de l'acte de langage comme élément central de sa théorie sociologique. Précisons que face aux attaques contre sa perspective critique initiale il a précisé sa position en s'acheminant vers une théorie normative de l'argumentation dans le cadre d'une pragmatique de l'action communicationnelle.

En s'inspirant de la cybernétique, Niklas Luhmann s'est de son côté également orienté vers la communication, mais d'une manière bien différente ${ }^{33}$. Après son "tournant autopoiétique ", et comme le montre le texte traduit pour ce volume, la communication devient la notion centrale de sa sociologie, et il reformule sa Funktion der Religion (1977) en Religion der Gesellschaft (paru deux ans après sa mort, en 2000 ; en anglais : A System Theory of Religion ${ }^{34}$, 2013). D’une manière générale, Luhmann réinterroge l'interprétation causaliste de l'action et en particulier le schéma fin-moyens en proposant une vue d'ensemble de la situation; il pose donc la même question que les pragmatistes comme Dewey ou Joas, mais apporte une réponse différente (Joas, 1999: 162-164). Le texte ici traduit, "La religion comme communication », permet d'entrer dans la pensée systémique de l'auteur qui aborde les religions comme des systèmes de sens constitués «de communication et non d'individus en train de communiquer » (E. Ferrarese, 2007 : 59).

Troisième témoignage de ce " tournant communicationnel ", la contribution de Luckmann, et plus largement de la tradition schutzienne, s'appuie sur George Herbert Mead, dont la notion de " communication » n'a d'ailleurs été transformée en « interaction » que plus tardivement par Herbert Blumer et d'autres. Le propos se fonde surtout sur l'étude empirique des processus de communication ${ }^{35}$ et développe une typologie comportant trois niveaux de transcendances. Les " petites transcendances » relient à ce qui est absent dans le temps ou dans l'espace (ce qui est derrière cette porte, ce que je ferai demain), les « moyennes » renvoient à ce que pense ou éprouve autrui, les «grandes " renvoient à des réalités non accessibles dans le monde de notre vie quotidienne (rêve, extase...). Son approche s'inspire des travaux de Schütz qu'il a édités après sa disparition, Die Strukturen der Lebenswelt (1989). Luckmann montre que, y compris dans ce qui est aujourd'hui considéré comme religieux, les «petites » et «moyennes » transcendances prennent plus d'importance, dans la sacralisation de l'individu ou l'importance de la réalisation de soi, et c'est pourquoi il parle de " rétrécissement de la transcendance ».

33. Luhmann a été l'élève de Parsons mais il est aussi influencé par la phénoménologie husserlienne.

34. Pour d'éclairantes balises (francophones) sur ce volume, voir Laflamme (2013); voir aussi : R. Laermans et G. Verschraegen (2001) et sur Funktion der Religion : T. Schöfthaler (1980).

35. L'école de Luckmann, dite aussi " école de Constance », est reconnue pour son approche ethnographique au sein de la recherche sociologique, pour ses analyses de la communication dans la vie quotidienne (par exemple à propos de conversions religieuses), qui recourent notamment aux enregistrements audio et vidéo. 
Hubert Knoblauch dans sa contribution à ce dossier reformule l'idée luckmannienne de "religion invisible » afin de prendre en compte la nouvelle visibilité du religieux. Il présente une théorie de la religion qui s’appuie sur la notion phénoménologique de transcendance, sur la sociologie de la connaissance de Schütz et Luckmann et sur une reformulation du constructivisme social de Berger et Luckmann en « constructivisme communicationnel ». Dans sa perspective, la transcendance doit être comprise comme une catégorie sociale essentielle de l' « agir communicationnel », qui s'applique à la religion et en particulier à la spiritualité ou la religion «populaire » contemporaine. Soulignons que le terme populaire est ici utilisé dans le sens de phénomènes largement répandus dans la société, transgressant les limites de la connaissance institutionnalisée et largement médiatisée. On est loin ici du sens discuté puis réfuté dans l'espace francophone des années 1970 (F.-A. Isambert, 1982).

Si la sociologie a produit de nombreux travaux sur la sécularisation, très peu de chercheurs se sont intéressés à la non-religion ${ }^{36}$, si ce n'est pour en faire une catégorie dans les enquêtes quantitatives. L'article de Monika Wohlrab-Sahr et Tom Kaden est à ce titre particulièrement novateur, en présentant une étude relationnelle de la religiosité, c'est-à-dire comment se construit le non-religieux face au religieux et avec quel type de conflictualités, dans deux contextes, en RDA et aux États-Unis. Les auteurs montrent que la référence à la science est centrale dans l'identité des non-religieux, mais avec des modalités différentes. En RDA, la non-religiosité était une norme sociale et la dévaluation de la religion par le politique s'appuyait sur la science. Aux États-Unis, la non-religiosité apparaît comme un non-conformisme (même si le nombre de personnes se déclarant sans religion a doublé dans la dernière décennie du $\mathrm{XX}^{\mathrm{e}}$ siècle) et les disputes sur le créationnisme font irruption aux marges des systèmes religieux et politiques. Le mimétisme scientifique des créationnistes a pour pendant un mimétisme religieux des non-religieux ${ }^{37}$.

Les deux derniers articles nous déplacent vers la macrosociologie. La contribution de Detlef Pollack, qui propose de mettre la sécularisation au banc d'essai des enquêtes quantitatives internationales, atteste de la vitalité d'un double débat: méthodes quantitatives versus qualitatives et sécularisation versus modernités (ou sécularités) multiples. L'auteur confronte ses hypothèses sur la modernité religieuse à des enquêtes menées en Europe et aux États-Unis sur la religion et défend la thèse de la sécularisation, tout en accordant une certaine validité à des thèses alternatives de transformation et d'individualisation du religieux.

36. Voir cependant S. Denèfle, 1997.

37. Cette demande d'être considéré comme une vision du monde parmi les autres est analogue à la prise en compte du non-religieux dans des cérémonies interreligieuses en France (par des poésies ou extraits de la Déclaration des droits de l'homme) (Lamine, 2004 : 187). 
Enfin l'article de Mathias Koenig s'inscrit dans une macro-sociologie comparative et d'inspiration wébérienne. Plusieurs auteurs posent en effet aujourd'hui la question de la pluralité des formes de modernité (S. Eisenstadt, 2007 ; M. WohlrabSahr et M. Burchardt, 2012) ou des formes de sécularité (T. Asad, 2003), soulignant que modernité et sécularité sont aussi des constructions culturelles ${ }^{38}$. Cet article propose une discussion originale des travaux de Shmuel Eisenstadt sur les «modernités multiples » en les faisant dialoguer avec l'approche néo institutionnaliste de John Meyer qui met l'accent sur la « signification culturelle universelle » de la rationalisation et pointe le degré élevé d'isomorphisme entre les États ou les organisations. S'appuyant sur divers exemples occidentaux et asiatiques, l'auteur montre l'importance d'être attentif aux cadrages culturels et aux contingences historiques pour analyser les nouveaux imaginaires du « séculier » et les variantes institutionnelles de la modernité.

Héritages et postérités de la sociologie allemande de la religion : ce dossier entend contribuer à un meilleur accès dans l'aire francophone à quelques «classiques " (Simmel, Luhmann, Luckmann), et à une meilleure connaissance de certaines perspectives contemporaines spécifiquement germaniques, l'ensemble de ces articles nous permettant aussi de réfléchir à ce qui nous différencie. De ces héritages intellectuels, l'analyse dépasse le cadre de notre exploration, mais nous en avons évoqué quelques traits : la place affirmée de la théorie et le tournant communicationnel. Ces héritages sont aussi historiques et géographiques : la biconfessionnalité, la situation de l'ex-RDA et la proximité de l'aire orthodoxe ont favorisé une attention plus marquée à la diversité des formes de modernités ou de sécularités.

Anne-Sophie LAMINE

Dynamiques Européennes

CNRS UMR 7367 - Université de Strasbourg anne-sophie.lamine@unistra.fr

Hubert KNOBLAUCH

Université technique de Berlin hubert.knoblauch@tu-berlin.de

\footnotetext{
38. Ces discussions sont globalement moins introduites en France (google scholar : 426 vs 91, pour Eisenstadt, cf. note 21) ; notons cependant Isambert (1992), Hervieu-Léger (2003) et Willaime (2006). La thématique est davantage traitée en termes de régulation du religieux, avec des approches tendanciellement différentes, soulignant les écarts (Willaime, 2010) ou au contraire la convergence des contextes nationaux (Portier, 2010).
} 


\section{Bibliographie}

BECKFORD James A., 2000, "Start Together and Finish Together": Shifts in the Premises and Paradigms Underlying the Scientific Study of Religion ", Journal for the Scientific Study of Religion, 39-4, p. 481-495.

Berger Peter, Luckmann Thomas, 1967, The Social Construction of Reality, New York, Anchor.

Bobineau Olivier, TANK-STORPer Sébastien, 2012, Sociologie des religions, Paris, Armand Colin.

ChIVA Isac, 1987, "Face à face ", Ethnologies en miroir : la France et les pays de langue allemande, in Chiva Isac, Utz Jeggle (dir.), Paris, Éditions de la MSH.

CiPRIANI Roberto, 2004, Manuel de sociologie de la religion, Paris, L'Harmattan.

DAIBER Karl-Fritz, LuCKMANn Thomas (dir.), 1983, Religion in den Gegenwartsströmungen der deutschen Soziologie, München, Chr. Kaiser [les versions anglaises ou françaises de la plupart des textes ont initialement été publiées in Social Compass, 1980, $27-1$ : Daiber, Drehsen, Fischer, Marhold, Lukatis, Krebber, Mörth, Schöfthaler, Seyfarth].

DAIBER Karl-Fritz, 1980, «Introduction », Social Compass, 27-1, p. 5-8.

DAviE Grace, 2004, "Creating an Agenda in the Sociology of Religion: Common Sources/ Different Pathways ", Sociology of Religion, 65-4, p. 323-340.

-, 2007, The Sociology of Religion, London, Sage.

DeNÈFLE Sylvette, 1997, Sociologie de la sécularisation : être sans-religion en France à la fin $d u X^{e}$ siècle, Paris, L'Harmattan.

Dianteill Erwan, Löwy Michael, 2005, Sociologies et religion: Approches dissidentes. II, Paris, Presses universitaires de France.

-, 2009, Sociologies et religion: Approches insolites. III. Paris, Presses universitaires de France.

DrehSEN Volker, 1980, « Bibliographie sélectionnée de sociologie de la religion et de l’Église en langue allemande après 1945 ", Social Compass, 27-1, p. 101-102.

-, 1980, «Dimensions of Religiosity in Modern Society. An Approach to a Systematical Stock-taking of Structural-Functionalist Analysis ", Social Compass, 27-1, p. 51-62.

EISENSTADT Shmuel N., 2007, "Une réévaluation du concept de modernités multiples à l'ère de la mondialisation ", Sociologie et sociétés, 39-2, p. 199-223.

Ferrarese Estelle, 2007, Niklas Luhmann, une introduction, Paris, Pocket/La Découverte.

FISCHER Wolfram, MARHOLD Wolfgang, 1980, «The Concept of Symbolic Interactionism in the German Sociology of Religion ", Social Compass, 27-1, p. 75-84.

FurSETH Inger, RePSTAD Pål, 2006, An introduction to the Sociology of Religion: Classical and Contemporary Perspectives, Surrey, Ashgate Publishing.

GABRIEL Karl, Reuter Hans-Richard (dir.), 2004, Religion und Gesellschaft, Paderborn, Schöningh.

GAlembert (DE) Claire de, 2004, « Die Religionssoziologie “à la française”. Vom positivistischen Erbe zur Erforschung der religiösen Moderne ", (Introduction) in HervieuLéger D., Pilger und Konvertiten, Würzburg, Ergon, p. IX-L.

GoldschmidT Dietrich, 1959a, "Standort und Methoden der Religionssoziologie ", in Soziologie und moderne Gesellschaft. Verhandlungen des 14. Deutschen Soziologentages vom 20-24 Mai 1959 in Berlin, Buch Alexander, Deutsche Gesellschaft für Soziologie (dir.), Stuttgart, Enke, p. 139-156. 
-, 1959b, "Die Religionssoziologie in der Bundesrepublik Deutschland ", Archives de sociologie des religions, 8 , p. 53-70 [une version légèrement modifiée du texte de 1959a].

HaCH Jürgen, 1980, Gesellschaft und Religion in der Bundesrepublik Deutschland, Heidelberg, Quelle \& Meyer.

Heilbron Johan, Guilhot Nicolas, Jeanpierre Laurent, 2009, «Vers une histoire transnationale des sciences sociales ", Sociétés contemporaines, 73-1, p. 121-145.

Helle Horst-Jürgen, 1997, Religionssoziologie. Entwicklungen der Vorstellungen vom Heiligen, München, Oldenbourg.

Hervieu-LéGer Danièle, 1998, "Les recompositions religieuses et les tendances actuelles de la sociologie des religions en France » Social Compass, 45-1, p. 143-153.

-, 1999, Le pèlerin et le converti, Paris, Flammarion [Pilger und Konvertiten, Würzburg, Ergon, 2004].

-, 2003, "Pour une sociologie des "modernités religieuses multiples" : Une autre approche de la "religion invisible" des sociétés européennes ", Social Compass 50-3, p. 287-295.

Hervieu-Léger Danièle, Willaime Jean-Paul, 2001, Sociologies et religion : approches classiques, vol. 1, Paris, Presses universitaires de France.

ISAMBERT François-André, 1982, Le sens du sacré. Fête et religion populaire, Paris, Éditions de Minuit, coll. "Le sens commun".

-, 1992, De la religion à l'éthique, Paris, Cerf, coll. « Sciences humaines et religions ».

JoAs Hans, 1999 [1992], La créativité de l'agir, Paris, Cerf.

KALBERG Stephen, 2004, "Difficultés d'un consensus transnational sur une théorie sociologique unifiée », Revue du MAUSS, 2, p. 173-188.

KeHRER Günter, 1968, Religionssoziologie, Berlin, Göschen.

-, 1988, Einführung in die Religionssoziologie, Darmstadt, Wissenschaftliche Buchgesellschaft.

KNoblauch Hubert, 1995, Kommunikationskultur. Die kommunikative Konstruktion kultureller Kontexte, Berlin, Walter de Gruyter.

-, 1999, Religionssoziologie, Berlin, Walter de Gruyter.

-, 2001, "Communication, Contexts and Culture. A Communicative Constructivist Approach to Intercultural Communication ", in Culture in Communication. Analyses of Intercultural Situations, di Luzio Aldo, Günthner Susanne, Orletti Franca (dir.), Amsterdam/Philadelphia: John Benjamins, p. 3-33.

-, 2003, Qualitative Religionsforschung Paderborn, Schöningh.

KitAGaWa Joseph, 1956, "Joachim Wach et la sociologie de la religion ", Archives de sociologie des religions, 1 , p. 25-40.

Koenig Matthias, Willaime Jean-Paul (dir.), 2008, Religionskontroversen in Frankreich und Deutschland, Hamburg, Hamburger Edition.

Koenig Matthias, Wolf Christof, 2013, "Religion und Gesellschaft. Aktuelle Perspektiven », Kölner Zeitschrift für Soziologie und Sozialpsychologie, 65-1, p. 1-23.

KRECH Volkhard, 1999, Religionssoziologie, Bielefeld, Transcript.

Laermans Rudi, Verschraegen Gert, 2001, "The Late Niklas Luhmann on Religion. An Overview ", Social Compass, 48-1, p. 7-20.

Laflamme Diane, 2013, "Balises pour une lecture croisée des textes de Luhmann sur la religion ", Canadian Journal of Sociology, 38-2, p. 255-267.

LAmine Anne-Sophie, 2004, La cohabitation des dieux, Paris, Presses universitaires de France.

Lassave Pierre, 2008, "Entre sociologie et anthropologie des religions ", Archives de sciences sociales des religions, 142, p. 151-167. 
LE Bras Gabriel, 1955 et 1956, Études de sociologie religieuse, 2 vol., Paris, Presses universitaires de France.

LuckManN Thomas, 1960, "Neuere Schriften zur Religionssoziologie ", Kölner Zeitschrift für Soziologie und Sozialpsychologie, 12-2, p. 315-326.

-, 1963, Zum Problem der Religion in der modernen Gesellschaft: Institution, Person und Weltanschaunng, Freiburg/Breisgau, Rombach.

-, 1967, The Invisible Religion. The Problem of Religion in Modern Society. New York, Macmillan.

Luckmann Thomas, Ferreux Jean, 2006, «Un entretien avec Thomas Luckmann », Sociétés, 3, p. 45-51.

Luhmann Niklas, 2013 [2000], A System Theory of Religion, Stanford, Stanford Univ. Press.

LUKATIS Ingrid, KREBBER Hartmut, 1980, « Recherches empiriques concernant la religion en Allemagne fédérale, en Autriche et en Suisse allémanique », Social Compass, 27-1, p. 85-100.

Lyman Stanford M., 1994, "A Haven for Homeless Intellects. The new school and its exile faculties » International Journal of Politics, Culture, and Society 7-3, p. 493-512.

MARY André, 2006, "Les archives... cinquante ans... après ", Archives de sciences sociales des religions, 4, p. 9-24.

MATTHES Joachim, 1967, Religion und Gesellschaft. Einführung in die Religionssoziologie I. Reinbek bei Hamburg, Rowohlt.

-, 1969, Kirche und Gesellschaft. Einführung in die Religionssoziologie II. Reinbek bei Hamburg, Rowohlt.

MAYER Carl, 1933, Sekte und Kirche. Ein religionssoziologischer Versuch, Heidelberg, Weiss.

MensChing Gustav, 1947, Religionssoziologie, Bonn, Röhrscheid.

Moebius Stephan, Lothar Peter, 2004, Französische Soziologie der Gegenwart, Konstanz, UVK.

Moebius Stephan, 2006, Die Zauberlehrlinge. Soziologiegeschichte des Collège de Sociologie (1937-1939), Konstanz, UVK.

MÖRTH Ingo, 1980, «La sociologie de la religion comme Théorie Critique (L’École de Francfort) ", Social Compass, 27-1, p. 27-50.

MüNCH Richard, 1991, "American and European Social Theory: cultural identities and social forms of theory production ", Sociological Perspectives, 34, p. 313-335.

PICKel Gert, 2011, Religionssoziologie. Eine Einführung in zentrale Themebereiche, Wiesbaden, VS Verlag für Sozialwissenschaften.

POLLAK Michael, 1988, "La place de Max Weber dans le champ intellectuel français ", Droit et société, 1988, 9, p. 195-210.

PORTIER Philippe, 2010, « Modernités plurielles ? Une approche longitudinale des modèles nationaux de régulation du croire dans les démocraties occidentales ", in Pluralisme religieux et citoyenneté, M. Milot, P. Portier, and J.-P. Willaime (dir.), Rennes, Presses universitaires de Rennes, p. 241-271.

QUÉRÉ Louis, 1991, "D’un modèle épistémologique de la communication à un modèle praxéologique ", Réseaux 9-46, p. 69-90. 
Scheler Max, 1926, Die Wissensformen und die Gesellschaft, Leipzig, Der neue Geist [Le premier des trois essais constituant ce volume a été traduit en français par Sylvie Mesure : Problèmes de sociologie de la connaissance, Paris, Presses universitaires de France, 1993].

SCHLUChTER Wolfgang, 2005, "Éléments d'un programme de recherche wébérien ", Revue française de sociologie, 46-4, p. 653-683.

SchnetTler Bernt, 2006, Thomas Luckmann, Konstanz, UVK, coll. "Klassiker der Wissenssoziologie ».

SCHÖFTHALER Traugott, 1980, "La théorie systémique dans la sociologie de la religion dans les pays de langue allemande. Les paradoxes religieux de Niklas Luhmann ", Social Compass, 27-1, p. 63-74.

SCHRECKER Cherry (dir.), 2012, Transatlantic Voyages and Sociology: The Migration and Development of Ideas, Surrey, Ashgate.

SchütZ Alfred, Luckmann Thomas, 1973 et 1989 [1974 et 1983], The Structures of the Life-World, vol. I et II, Evanston, Northwestern Univ. Press.

SÉGUY Jean, 1970, "Sociologie de la connaissance et sociologie des religions ", Archives de sociologie des religions, 30, p. 91-107.

Seyfarth Constans, 1980, "The West German Discussion of Max Weber's Sociology of Religion since the 1960s ", Social Compass, 27-1, p. 9-25.

STARK Werner, 1974, Grundriß der Religionssoziologie, Freiburg, Rombach.

STichweH Rudolf, 1999, "Niklas Luhmann ", in Klassiker der Soziologie, T. 2, Von Talcott Parsons bis Pierre Bourdieu, Kaesler Dirk (dir.), München, C.H. Beck 1999, p. 206229 [version anglaise de ce texte: fiw.uni-bonn.de/fiw-start/demokratieforschung/ personen/stichweh/pdfs/81_stw_niklas-luhmann-blackwell-companion-to-major-socialtheorists.pdf, consulté le 16 mai 2014].

Trom Danny, 2008, « La crise de la critique sociale, vue de Paris et de Francfort ", Esprit, 7, p. 108-126.

TSCHANNEN Olivier, 1990, «Le débat sur la sécularisation à travers les Actes de la CISR », Social Compass, 37-1, p. 75-84.

Tyrell Hartmann, 1995, "Von der "Soziologie statt Religion” zur Religionssoziologie ", in Religionssoziologie um 1900, Tyrell Hartmann, Krech Volkhard (dir.), Würzburg, Ergon, p. 79-127.

Tyrell Hartmann, Krech Volkhard, KNoblauch Hubert (dir.), 1995, Religion als Kommunikation, Würzburg, Ergon.

WaCH Joachim, 1931, Einführung in die Religionssoziologie, Tübingen, Mohr, [trad. fr. Sociologie de la religion, Paris, Payot, 1955].

Wallisch-Prinz Bärbel, 1977, Religionssoziologie. Eine Einführung, Stuttgart, Kohlhammer.

Watier, Patrick, 1996, "G. Simmel : religion, sociologie et sociologie de la religion ", Archives des sciences sociales des religions, 93-1, p. 23-50.

-, 2008, «Erkenntnisinteressen der klassischen Religions-soziologie: Durkheim, Simmel, Weber », Religionskontroversen in Frankreich und Deutschland, Köenig Matthias, Willaime Jean-Paul (dir.), Hamburg, Hamburger Edition, p. 206-238. 
Willaime Jean-Paul, 1995, Sociologie des religions, Paris, Presses universitaires de France, coll. "Que sais-je ?».

-, 1999, "French-language Sociology of Religion in Europe since the Second World War ", Schweizerische Zeitschrift für Soziologie/Revue suisse de sociologie, 25-1, p. 343-371.

-, 2001, « Max Weber » et " Georg Simmel », in Sociologies et religion : approches classiques, vol. 1, Hervieu-Léger D., Willaime J.-P. (dir.), Paris, Presses universitaires de France, p. 59-110 et 111-146.

-, 2004, "The Cultural Turn in the Sociology of Religion in France ", Sociology of Religion, 65-4, p. 373-389.

-, 2006, "La sécularisation : une exception européenne ? ", Revue française de sociologie, 47-4, p. 755-783.

-, 2010, "Pluralité religieuse et citoyenneté en France et en Allemagne ", in Pluralisme religieux et citoyenneté, M. Milot, P. Portier, and J.-P. Willaime (dir.) Rennes, Presses universitaires de Rennes, p. 139-152.

Wohlrab-SAHr Monika, 2000, "What has happened since Luckmann 1960? Sociology of religion in Germany, Austria, and Switzerland ", Schweizerische Zeitschrift für Soziologie, 26-1, p. 169-192.

Wohlrab-SAHr Monika, Burchardt Marian, 2012, "Multiple Secularities: Towards a Cultural Sociology of Secular Modernities », Comparative Sociology, 11-6, p. 875-909. 